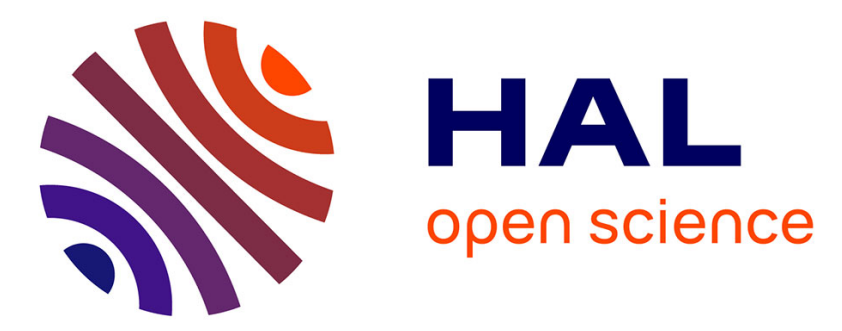

\title{
Variations of the Electron Fluxes in the Terrestrial Radiation Belts Due To the Impact of Corotating Interaction Regions and Interplanetary Coronal Mass Ejections
}

\author{
R. Benacquista, D. Boscher, S. Rochel, V. Maget
}

\section{To cite this version:}

R. Benacquista, D. Boscher, S. Rochel, V. Maget. Variations of the Electron Fluxes in the Terrestrial Radiation Belts Due To the Impact of Corotating Interaction Regions and Interplanetary Coronal Mass Ejections. Journal of Geophysical Research Space Physics, In press, 10.1002/2017JA024796 . hal-01728877

\section{HAL Id: hal-01728877 \\ https://hal.science/hal-01728877}

Submitted on 12 Mar 2018

HAL is a multi-disciplinary open access archive for the deposit and dissemination of scientific research documents, whether they are published or not. The documents may come from teaching and research institutions in France or abroad, or from public or private research centers.
L'archive ouverte pluridisciplinaire HAL, est destinée au dépôt et à la diffusion de documents scientifiques de niveau recherche, publiés ou non, émanant des établissements d'enseignement et de recherche français ou étrangers, des laboratoires publics ou privés. 


\title{
Variations of the electron fluxes in the terrestrial radiation belts due to the impact of Corotating Interaction Regions and Interplanetary Coronal Mass Ejections
}

\author{
R. Benacquista ${ }^{1}$, D. Boscher ${ }^{1}$, S. Rochel ${ }^{1}$, V. Maget $^{1}$ \\ ${ }^{1}$ Département Physique, Instrumentation, Environnement, Espace, ONERA, Toulouse, France
}

\section{Key Points:}

- Around $L^{*}=3$, CIRs can increase the flux of $>30 \mathrm{keV}$ to $>100 \mathrm{keV}$ electrons but no for higher energies (unlike ICMEs).

- The maximal ratio between the flux post- and pre-event is greater than 100 , is found around $L^{*}=3$ and is caused only by a few ICMEs.

- The intensity of magnetic storms, defined with the minimum value of the Dst index, is linked no only to the minimal value of $L^{*}$ for which the flux increase but also to the amplitude of this increase.

Corresponding author: R. Benacquista, remi . benacquista@onera.fr 


\begin{abstract}
In this paper, we study the variations of the radiation belts electron fluxes induced by the interaction of two types of solar wind structures with the Earth magnetosphere: the corotating interaction regions and the interplanetary coronal mass ejections. We use a statistical method based on the comparison of the pre- and post-events fluxes. Applied to the NOAA-POES data, this gives us the opportunity to extend previous studies focused on relativistic electrons at geosynchronous orbit. We enlighten how CIRs and ICMEs can impact differently the electron belts depending on the energy and the $L_{\text {shell }}$. In addition, we provide a new insight concerning theses variations by considering their amplitude. Finally, we show strong relations between the intensity of the magnetic storms related to the events and the variation of the flux. These relations concern both the capacity of the events to increase the flux and the deepness of these increases.
\end{abstract}

\title{
1 Introduction
}

The radiation belts are a region in the near earth space environment formed by trapped and highly energetic particles. This region is harmful for the great number of satellites orbiting in and several anomalies have been reported on board, ranging from a temporary gap in the instrumental data to a total failure of the spacecraft (see Table 1 in Cannon [2013]).

The Interplanetary Coronal Mass Ejections (ICMEs) and the Corotating Interaction Regions (CIRs) are known as the main structures of the solar wind able to perturb the magnetosphere and cause geomagnetic storms of various profiles and intensities [Borovsky and Denton, 2006; Alves et al., 2006; Turner et al., 2006; Richardson and Cane, 2011; Benacquista et al., 2017]. The coupling between the solar wind and the magnetosphere also implies variations of the fluxes in the radiation belts over several orders of magnitude.

These variations have been previously studied, especially for relativistic electrons at geosynchronous orbit, using two statistical methods: the Superposed Epoch Analysis (SEA) and the comparison of pre- and post-storm levels of flux. Reeves et al. [2003] and Anderson et al. [2015] used the LANL spacecraft and applied the pre/post-storm levels comparison method to the electron flux $>2 \mathrm{MeV}$. They set their list of events with respect to the $D s t$ index by looking for any geomagnetic storms moderate or intense (for Reeves et al. [2003]) and weak (for Anderson et al. [2015]). They found that any magnetic storms 
could cause either a decrease, an increase or no significant variation of the flux. The proportions are such (respectively 19\%, 53\%, 28\% for Reeves et al. [2003] and 32\%, 42\%, $26 \%$ for Anderson et al. [2015]) that these results underline the unpredictability of the reaction of the belts, at least for this energy $(>2 \mathrm{MeV})$, and this orbit (geosynchronous).

To go further, other authors sorted the geomagnetic storms depending on their cause (CIRs or ICMEs). Miyoshi and Kataoka [2005] applied SEA on relativistic (> 2MeV) electron flux, measured by GOES spacecraft. They found that CIRs cause much higher increases of the flux than ICMEs at the geosynchronous orbit. In addition, they separated the ICMEs in two groups depending on the intensity of the related magnetic storms (defined with the minimal value of $D s t$ ) and they found no significant difference. This again indicates that the intensity of the magnetic storm is not one of the relevant features to understand the variations of the flux at this energy and orbit (also confirmed by Kataoka and Miyoshi [2006]).

Other studies used the so-called multi-epoch SEA. By setting the epoch times relatively to the structure of the events (instead of the magnetic storms), they aimed to understand the contribution of each sub-structure on the variations of the flux. Kilpua et al. [2015] applied both multi-epoch SEA and pre-/post-event comparison to CIRs and ICMEs for the flux of relativistic electrons measured by GOES spacecraft. Their results confirm that CIRs are the most efficient structures to increase the flux at geosynchronous orbit. They showed that the flux decreases from the onset of the CIR to the stream interface and, then, increase from the crossing of the stream interface, during the remaining part of the CIR and the following high speed stream. Concerning the events related to the ICMEs, the upstream sheaths trend to make the flux decrease while the tendency for the ICMEs remains unclear. However, by sorting the events depending on the speed of the downstream solar wind, they emphasized the key role of high speed streams (following CIRs and ICMEs) to increase the flux of relativistic electrons at geosynchronous orbit.

These conclusions confirm other previous studies. Hietala et al. [2014] showed that the depleting effect of the sheath comes from the combination of the pressure increase, allowing a loss of electrons by magnetopause shadowing, and the increase of the ULF waves, pushing the particles to lower $L^{*}$ value by radial diffusion. According to Miyoshi and Kataoka [2008] and Miyoshi et al. [2013], the fast stream appears to be a necessary 
but insufficient condition for the increase of the flux. They showed that a negative $B_{Z}$ during the high speed stream is also necessary.

Therefore, this set of studies provides a good overview of the effects of the CIRs and the ICMEs on the evolution of relativistic electrons flux at geosynchronous orbit. However, these results are limited to only one energy range and one orbit and, thus, give an incomplete view of the dynamics of the radiation belts.

Recent studies using the Van Allen Probes data investigate the flux at other energies and $L^{*}$ values. Reeves et al. [2016] observed the radiation belts after seven events and highlighted their typical structure. They showed a strong dependence of the radiation belts dynamics to the energy and $L^{*}$ value. Turner et al. [2015] performed a statistical study by using the pre-/post-event comparison on a list of 52 events causing moderate or strong magnetic storms. They confirmed the strong dependencies to the energy and $L^{*}$ and showed that, regardless of the energy, the region between $L^{*}=3$ and $L^{*}=4.5$ is the most likely to make the fluxes increase. However, they defined their events by observing the variations of the Dst index and, therefore, did not differentiate the effect of the CIRs and the ICMEs while Kilpua et al. [2015], using the Van Allen Probes for three study cases (one SIR during March 2013, one ejecta in July 2013 and one sheath+ejecta in June 2013), observed that the radiation belt's response is clearly different according to the type of events. This trend was already observed by Kataoka and Miyoshi [2006] who performed SEA on the flux measured by NOAA-POES.

All the previous studies consider only the events related to magnetic storms with intensity of at least Dst $=-50 n T$ (except for Anderson et al. [2015]). However, recent studies also using the Van Allen Probes data show that the variations of the flux can occur during non-storm times. Through three use cases the authors show that events without related magnetic storms can also cause increases [Su et al., 2014, 2015] or decreases [Su et al., 2016] of the electrons flux over more than one order of magnitudes.

Despite the full covering of $L^{*}$ values and the several energy channels, the statistical studies using the Van Allen Probes data are limited because of the relatively small period during which the data are available (since 2012) and the particularly quietness of solar cycle 24 . In this study, we used the pre-/post-event comparison method to study the impact of the solar wind structures on the radiation belts. By using NOAA-POES data, we benefit from a very great length of time, for the entire range of $L^{*}$ and several energy channels. 
In addition, we used different lists of events, allowing the distinction between CIRs and ICMEs.

\section{Data and Methods}

We used the flux measured by the NOAA-POES spacecraft from POES-15 to POES19 covering the period since July 1998. Previous satellites have not been considered because of a change of the detectors (and their orientation) between POES-14 and POES-15. Each satellite are equipped with the SEM/MEPED instrumentation providing the electron flux for three energy channels : $>30 \mathrm{keV},>100 \mathrm{keV}$ and $>300 \mathrm{keV}$. However, because of strong contamination, one of the protons channel is commonly used to detect relativistic electrons $>1 \mathrm{MeV}$ [Yando et al., 2011]. For every time steps of one hour and every energy channels, we determined the flux for each value of $L^{*}$ between 1.0 and 8.0 with a resolution of $\Delta L^{*}=0.1$. If several satellites provide a value for the same time and $L^{*}$, we simply computed the geometrical mean value. Then, we applied a $24 \mathrm{~h}$ sliding window average on the flux. By doing so, we erased the variations of the flux due to orbital effects and MLT-dependencies for the low energy channels.

Since we aim to differentiate the impact of CIRs and ICMEs, we used existing lists of events. Most of the studies systematically consider the events with $D s t<-50 n T$ and set apart the weaker ones. Since one of their main conclusion is that the variations of the relativistic electron flux do not depend on the intensity of the related magnetic storms (at least for geosynchronous orbit), one might expect that a non-negligible number of weaker events would have cause substantial variations of the flux. As a consequence, the authors removed from their lists some events that could have been efficient from the radiation belts point of view. Therefore, we chose to use some lists of events based exclusively on the observation of the solar wind parameters. Several of these lists are available and we chose to use the lists from the studies of Mitsakou and Moussas [2014] (for the ICMEs) and Jian et al. [2006] (for the CIRs). The list of ICMEs provides 325 events between 1996 and 2008 using the OMNI data base. Since the flux data are available only since July 1998, this list is reduced to 278 events. For each event, the list provides the time of beginning of the disturbance upstream the ICME, the beginning of the ICME and the end of the ICME. The boundaries of the ICMEs are determined by looking for their typical signature in the plasma and the magnetic field. The list of CIRs originally provided some events from 1995 to 2004. It has later been completed up to 2009, and it provides 574 events between 
1995 and 2009 (440 since July 1998). Three dates, determined at the Lagrangian point L1, are provided for each event: the onset of the CIR, the stream interface and the end of the CIR. They are essentially determined with the observation of the total perpendicular pressure.

In this study, we applied the method of comparison of the flux pre-/post-events. This method has been widely used in the previous cited literature. It involves comparing the levels of flux before and after each event without considering their variations during the interaction interval between the structure and the magnetosphere. It can be summarized as follow: the levels of flux pre-events and post events are determined, then the ratio of both level are computed. If this ratio is lower than 0.5 , the event is considered to significantly decrease the flux. If the ratio is higher than 2.0, the flux is considered to have increased. Otherwise (between 0.5 and 2.0), there is no significant variation. Following Kilpua et al. [2015], we chose to determine the pre-events flux as the mean value between 36 hours and 12 hours before the onset of each event regardless of the energy. For the post-storm level, we looked for the average flux between $0 h$ and $24 h$ for the two lowest energy channels (>30keV and $>100 \mathrm{keV}$ ) and between $36 \mathrm{~h}$ and $60 \mathrm{~h}$ for the two highest energy channels ( $>300 \mathrm{keV}$ and $>1 \mathrm{MeV}$ ). Theses values have been chosen to avoid underestimation of the number of events increasing the flux.

\section{Results}

\subsection{Capacity of the CIRs and the ICMEs to increase or decrease the fluxes}

Based on the definition given in Section 2, figure 1 gives the percentage and number of events that lead to an increase, a decrease or no change of the flux as a function of the energy (color code), $L^{*}$ (x-axis), and the type of events (CIRs on the left panel, ICMEs on the right panel). For each plot, the area between the bottom of the graph and the dashed line corresponds to the events that decrease the flux. The area between the dashed line and the solid line corresponds to the events that cause no change of the flux and the area between the top of the graph and the solid line corresponds to the events that increase the flux. As an example, for the ICMEs (left panel) and the electrons $>30 \mathrm{keV}$ (green line) at $L^{*}=5,17 \%$ of the events of our list decrease the flux while $34 \%$ increase it.

First, we compared our results to those of other studies for relativistic electrons in geosynchronous orbit ( $\left.L^{*}=6\right)$ : Reeves et al. [2003]; Anderson et al. [2015]; Kilpua et al. 


\section{ICMEs}

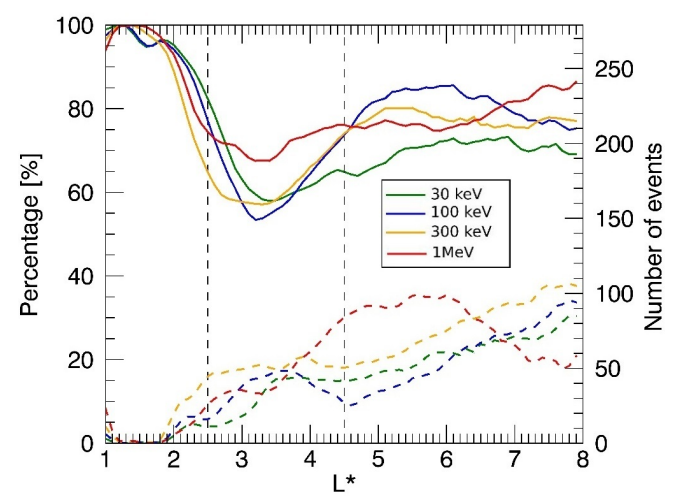

CIRs

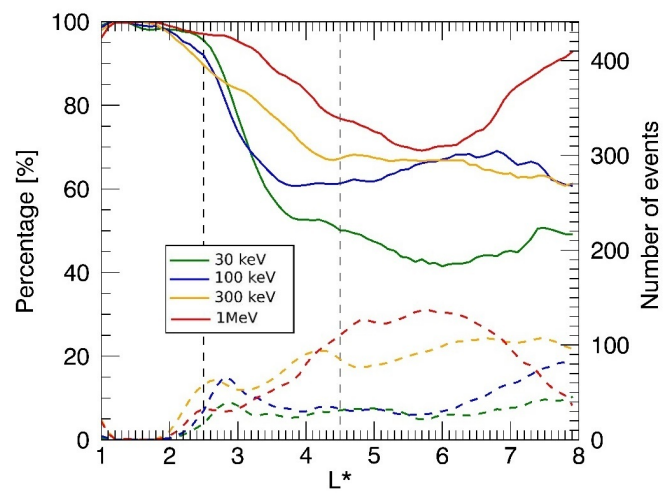

Figure 1. Percentage and number of events that make the flux increase or decrease as a function of $L^{*}$ (X-axis), the energy (color code), the type of events (ICMEs on the left panel, CIRs on the right). The dashed lines indicate the percentage (and number) of event resulting in a decrease of the flux and the solid lines correspond to the events resulting in a decrease or no change. Therefore, the area from the top to the solid line indicate the statistic of the events resulting in an increase of the flux.

[2015]; Turner et al. [2015]. The comparison indicates strong differences between the results of all studies. Turner et al. [2015] explained their differences of results with the study of Reeves et al. [2003] with the different period chosen, their study covering only a part of the quiet solar cycle 24 . In our case, we found systematically a lower proportion of events resulting in an increase of the flux and a higher proportion resulting in no change. In particular, the strong conclusion found by Kilpua et al. [2015] that CIRs are much more efficient than ICMEs to increase the flux at geosynchronous orbit is less clear in our results. From our point of view, the main explanation to such differences comes from the choice of the lists of events. Every studies base their statistics on a different list of events set with different criteria. In particular, Reeves et al. [2003], Turner et al. [2015] and Kilpua et al. [2015] considered only the events causing magnetic storms with $D s t<-50 n T$. The list we chose contain every events detected at point L1 regardless of the intensity of the related magnetic storms. Therefore, one might think that our list implies a consequent amount of non-effective events explaining why we found a higher proportion of events resulting in no change. However, by comparing the number of events rather than the percentages, we found that far more CIRs $(\approx 130)$ cause an increase of the 
flux than ICMEs $(\approx 70)$. Therefore, from the radiation belts point of view, an increase of the flux at geosynchronous orbit is due to a CIR almost two over three times.

In addition, our results provide indications for other energies and $L^{*}$ values. First, for the ICMEs, there is a range of $L^{*}\left(L^{*}=2.5-4.5\right)$ for which the ICMEs are particularly efficient to increase the flux. This, enlighten on the figure with two vertical dashed lines, is the same range regardless of the energy despite that the percentage is strongly reduce for relativistic electrons. On the other hand, the percentage of events leading to a decrease of the flux is higher when the energy and $L^{*}$ increase. Therefore, one can subdivide the belts in three regions as a function of the impact of the ICMEs: in the deepest zone $\left(L^{*}<\right.$ 2.5), ICMEs almost never cause variations of the flux (at least, for the events of our list). Between $L^{*}=2.5$ and $L^{*}=4.5$, they are efficient to make the flux increase while the opposite trend is observed above $L^{*}=4.5$.

The impact of the CIRs includes both similarities and differences with the ICMEs. First, the CIRs cannot impact the belts as deep as the ICMEs. This difference, although significant, is weak for the two first energy channels $(>30 \mathrm{keV}$ and $>100 \mathrm{keV})$. Then it increase for the upper energy channels. Contrarily to the ICMEs, the lowest $L^{*}$ impacted by the CIR depends on the energy. For instance at $L^{*}=3.5$, a large proportion of CIRs (42\%) increase the flux of electron $>30 \mathrm{keV}$ while this percentage fall down to $11 \%$ for the electrons $>1 \mathrm{MeV}$. Concerning the decrease of the flux, we notice the striking inability of the CIRs to decrease the flux for the lowest energy channels (> $30 \mathrm{keV}$ and $>100 \mathrm{keV})$.

These results clearly show the variability of the reaction of the radiation belts depending on the structure impacting, but also the $L^{*}$ and the energy. Therefore they can be compared with those of the study of Turner et al. [2015]. As they pointed out, a strong majority of the events of their list (only events with $D s t<-50 n T$ ) increase the flux in the region $L^{*}=3.5-4.5$. These results are confirmed by our study and we provide a clarification on the contribution of the ICMEs and the CIRs. For $>30 \mathrm{keV}$ and $>100 \mathrm{keV}$, both CIRs and ICMEs cause these increases. In contrast, for $>300 \mathrm{keV}$ and $>1 \mathrm{MeV}$, the CIRs are much less efficient (for these $L^{*}$ ) and almost only ICMEs can increase the flux. In addition, Turner et al. [2015] found that almost no event of their list were able to increase the flux of electrons $>1 \mathrm{MeV}$ for $L^{*}=2.5-3.0$. Our results are quite different as we show that $\approx 25 \%$ or the ICMEs of our list increase the flux for the similar energy 
range. This difference clearly comes from the considered time range. By using the RBSP data, Turner et al. [2015] were limited in a period during solar cycle 24 where nearly no events were strong enough to increase the flux at such $L^{*}$. By using NOAA-POES data, our study covers the solar cycle 23 with a consequent number of strong events. Concerning the decreases, ICMEs are clearly more efficient, especially for the low energy channels and it can be seen that the number and proportion of ICMEs that decrease the flux progressively increase with $L^{*}$.

This section dealt with the ratio of events causing significant variations of the flux depending on the energy, $L^{*}$ and the type of structure. However we did not discuss the amplitude of these variations. This is what we propose to do next section since it seems fair to differentiate an event doubling the flux from another one multiplying it by ten or more. Therefore, we will sort the events depending on the amplitude of these variations.

\subsection{Amplitudes of the variations of the flux}

We computed the ratio $R$ between the post and pre-event fluxes and classified the events within ten ranges of $R(R<0.01, R=0.01-0.1, R=0.1-0.2, R=0.2-0.5$, $R=0.5-1.0, R=1-2, R=2-5, R=5-10, R=10-100, R>100)$.

The results are displayed in figure 2 for the two types of structures and two energy channels $(>30 \mathrm{keV}$ and $>1 \mathrm{MeV})$. Each panel corresponds to one energy channel as indicated overhead and one structure as noted on the left side. The figures corresponding to the two other energy channels $(>100 \mathrm{keV}$ and $>300 \mathrm{keV}$ ) are given in figure 1 in the supporting information. On each panel, the area between two lines corresponds to the percentage and number of events related to the corresponding ratio. The grey area indicates the ratios $R=0.5-1.0$ and $R=1-2$ and therefore corresponds to the "no change" statistics in the previous section. As an example, at $L^{*}=4$ around $9 \%$ of the ICME increase the flux of electron $>30 \mathrm{keV}$ with a ration $R=5-10$.

For the flux $>30 \mathrm{keV}$, the most striking observation is the asymmetry between the amplitudes of increase and decrease. Indeed, both CIRs and ICMEs can cause increases much stronger than decreases since this is not unusual that the flux increase with $R>5$ while the decreases are almost only with $R<5$. It is also interesting to note that both ICMEs and CIRs can increase the flux with a ratio between 10 and 100 despite a different 

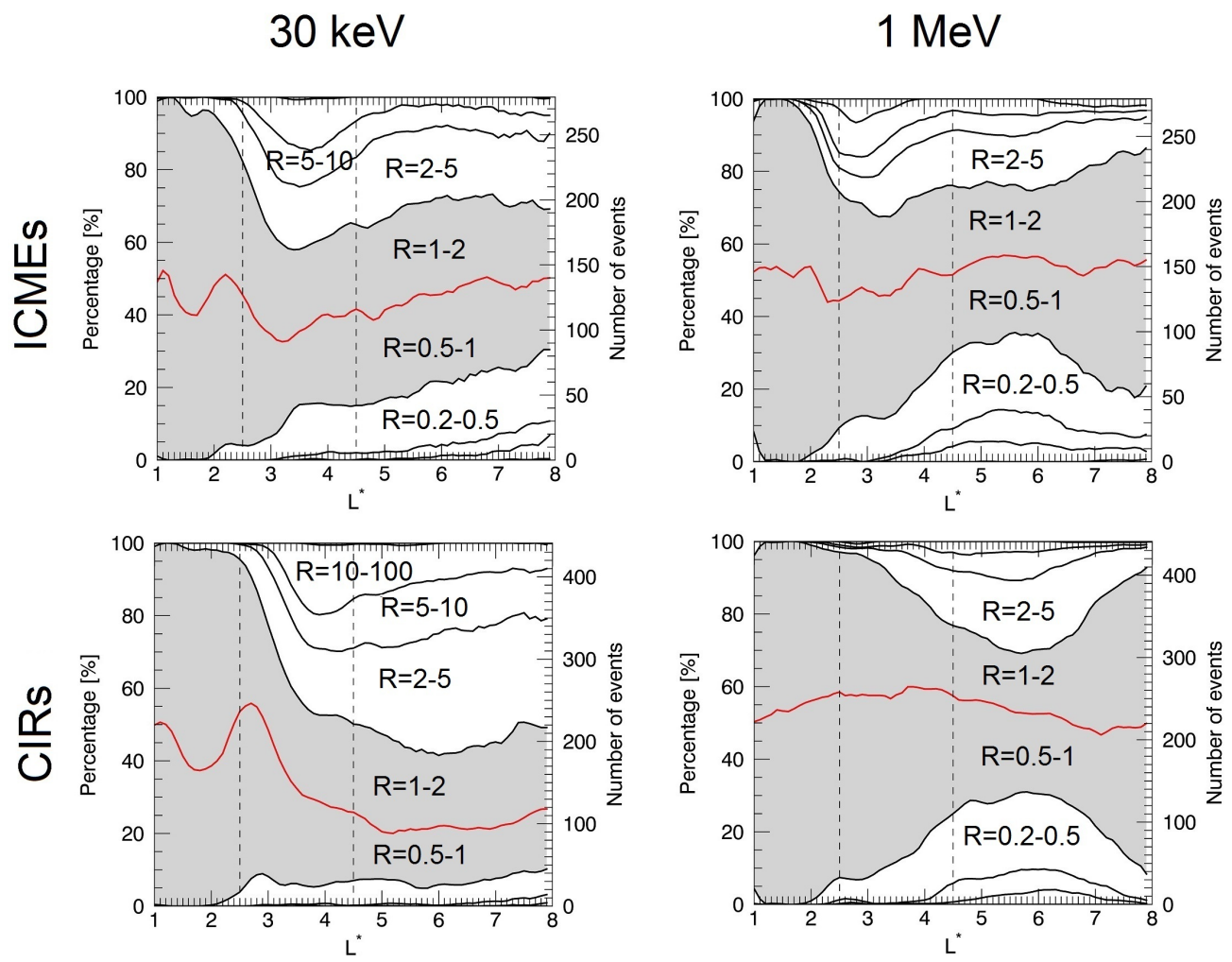

Figure 2. Distribution of the ICMEs (top panels) and the CIRs (bottom panels) for two energy ranges ( $>\quad 30 \mathrm{keV}$, left panels and $>1 \mathrm{MeV}$, right panels) depending on the ratio $R$ between the flux after and before the events. The red line distinguish the events that make the flux increase or decrease and the grey area enlightens the events corresponding to "no change" in the previous section $(\mathrm{R}=0.5-2)$

dependency on $L^{*}$. A few ICMEs can increase the flux with such ratios a bit deeper than CIRs but, on the contrary, for $L^{*}>5$, only CIRs can increase the flux with $R>5$.

For the flux $>1 \mathrm{MeV}$, the results are very different. Unlike the CIRs, a few ICMEs are able to cause intense increases of the flux $(R>100)$ on a limited range of $L^{*}$. On the other hand, the decreases seems to occurs with higher ratio even if the number of corresponding events is quite low. One can also notice that, only ICMEs can increase the flux with a ratio $R>5$ below $L^{*}=3.5$.

As showed in the previous section, the choice of the list of events can significantly impact the results of the statistical study. Following the same method, we computed the statistics on the list of events from Kilpua et al. [2015]. The results are given in figure 2 and 3 in the supporting information. As expected, the numerical values change strongly 
since the list of Kilpua et al. [2015] contain much less events associated to "no change" but one can observe that the variations and the dependencies in energy, $L^{*}$ and structure are preserved.

Despite we showed that the reaction of the radiation belts is statistically clearly different depending on the type of structure, our results suggest that two events (of the same type) can have very different effect. Therefore, it would be interesting to determine some feature of the events allowing to discriminate the reaction of the belts. In the next section, we propose to sort the events according to the intensity of the related magnetic storms.

\subsection{Reaction of the belts depending on the intensity of the related magnetic storms}

We consider now all 718 events (ICMEs and CIRs) and we sort them according to the intensity of the related magnetic storms (defined with the minimal value of the Dst index). We show on figure 3 the number of events increasing or decreasing the flux depending on this criteria. Each panel correspond to one energy channel and the statistics for each subgroup are given with different colors.

At geosynchronous orbit $\left(L^{*} \approx 6\right)$, we found that the number of events that increase or decrease the flux does not depend on the intensity of the related magnetic storm. This support the conclusion of previous studies for electrons $>1 \mathrm{MeV}$, and allow to extend it to other energy channels.

Our results also show that, in contrast, in the inner part of the belts, the intensity of the storms have a strong impact on the reaction of the belts. First, the more intense the magnetic storm is, the more likely it is to increase the flux. The probability to increase the flux, indeed increase progressively through the different subsets and almost every events related to a magnetic storm with $D s t<-150 n T$ increase the flux for $L^{*}=2.5-3.0$.

The intensity of the magnetic storm is not only linked to the probability to increase the flux but also to the deepness of this increase. For instance, for electrons $>30 \mathrm{keV}$ (upper left panel), events related to $D s t>-30 n T$ (green line) never increase the flux below $L^{*}=3$ while the most intense magnetic storms (orange and red lines) can increase the flux at least from $L^{*}=2$.

In previous studies, Su et al. [2014, 2015, 2016] showed that non-storm time events can cause variations of the electrons flux. Our results confirm that in the outer part of the 

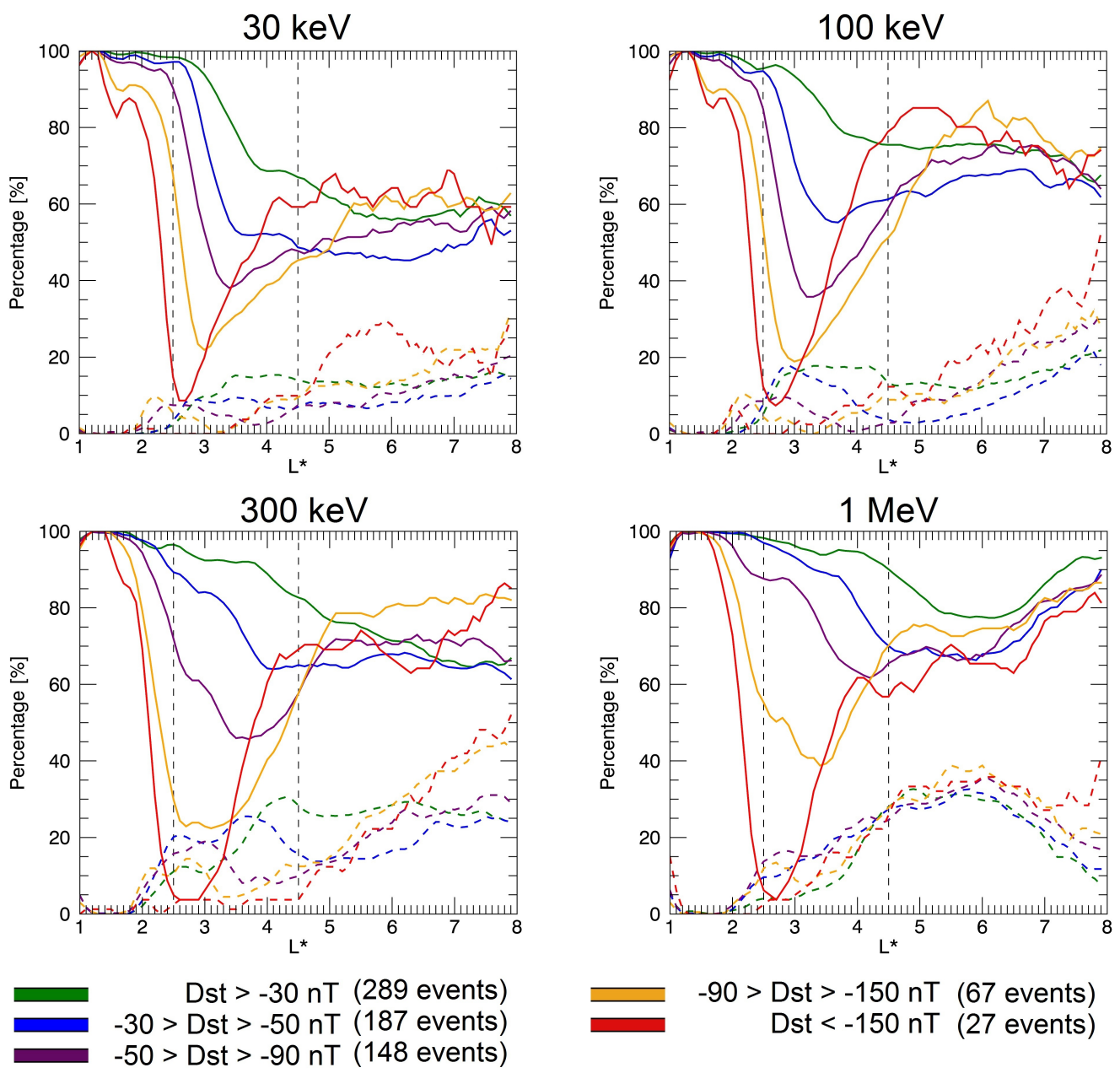

Figure 3. Percentage and number of events that make the flux increase or decrease as a function of $L^{*}(\mathrm{X}-$ axis), the intensity of the related magnetic storm (color code), for the different energy channels. The dashed lines indicate the percentage (and number) of event resulting in a decrease of the flux and the solid lines correspond to the events resulting in a decrease or no change. Therefore, the area from the top to the solid line indicate the statistic of the events resulting in an increase of the flux.

belts $\left(L^{*}>5\right)$, non-storm events, as well as more intense events, can cause variations of the flux. In the other hand, for $L^{*}<5$, our results indicate that non-storm events (green lines, $D s t>-50 n T)$ barely cause variations of the flux. In addition, the figure 4 gives the ratio $R$ between the post and pre-event fluxes corresponding to the 289 non-storm events for electrons $>1 \mathrm{MeV}$. Therefore this figure is similar to the right panels of figure 2 but only for non-storm events. 


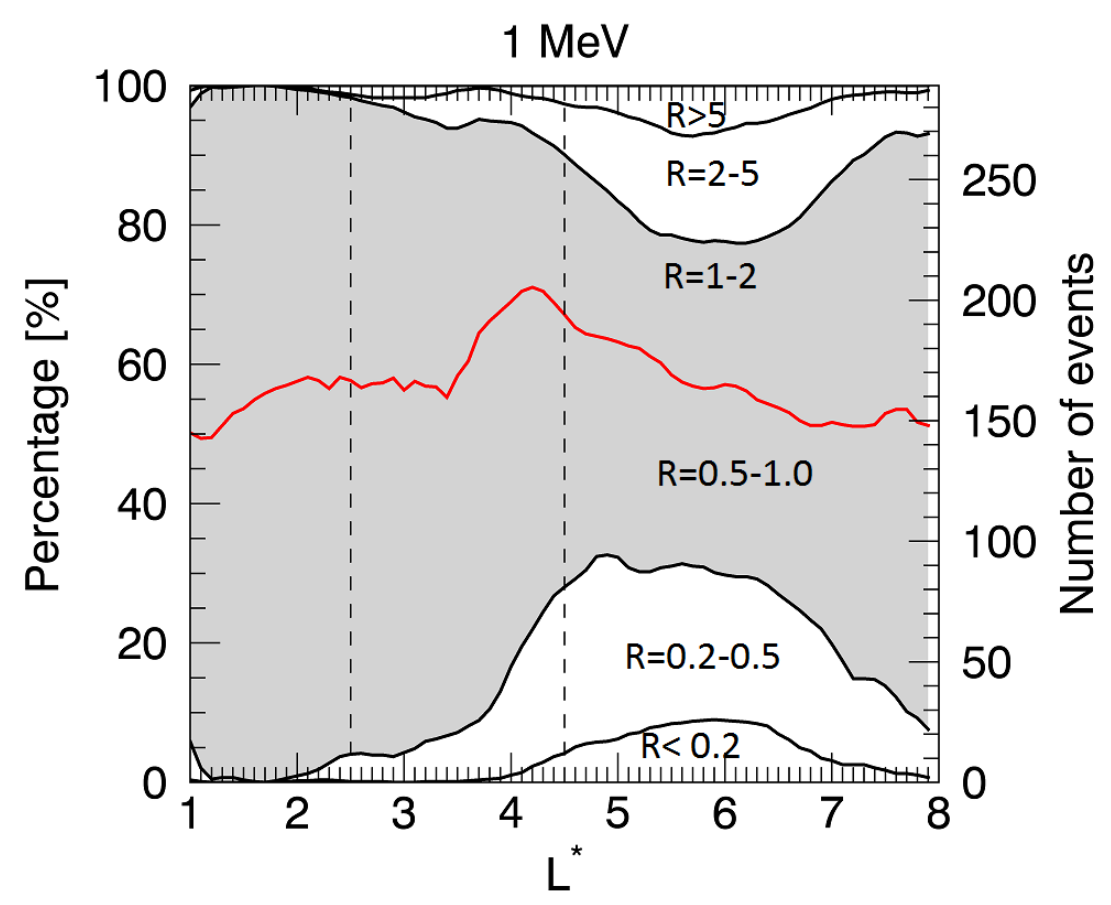

Figure 4. Ratio $R$ between the flux after and before the events for 289 non-storm events and depending on the $L^{*}$ parameter. These results correspond to the energy chanel $>1 \mathrm{MeV}$.The red line distinguish the events that make the flux increase or decrease and the grey area enlightens the events corresponding to "no change" in the previous section $(\mathrm{R}=0.5-2)$.

It can be seen that around $85 \%$ of these events cause variations (increases or decreases) with a ratio lower than 5. Therefore, the variations of the flux related to nonstorm events are not only infrequent but also limited to weak ratios whereas stronger events can increase the flux with a factor above 100 at lower $L^{*}$.

\section{Discussion}

Our statistical study clearly shows that the reaction of the belts is strongly dependent at the same time to the structure the event (CIR or ICME), to the energy and to the $L^{*}$ parameter. The variations of the flux are caused by a balance between different physical processes leading to the transport of the electrons, their gain or loss of energy and / or their total loss. Schematically, one can consider 3 zones in the belts. In the inner part (low $L^{*}$ ), loss process dominate due to pitch angle scattering caused by the interaction between Hiss waves and particles. In the intermediary part of the belts, acceleration processes dominate thanks to the simultaneous contribution of radial diffusion and the interaction of the elec- 
trons with Chorus waves. In the outer part of the belts, the magnetopause shadowing lead to strong and efficient loss of particles. Of course, the boundary between theses regions depend on each event that cause the intensification of theses processes in different proportions. The position of the plasmapause can provide an indication of the boundary between the two first zones because Hiss and Chorus waves are preferably generated respectively inside and outside the plasmasphere. In the other hand, the position of the magnetopause allows to estimate the regions where the magnetopause shadowing occurs.

In the figure 5, we plot the sum curves corresponding to the position of the plasmapause (left panel) and the magnetopause (right panel) during the events. The position of the plasmapause is computed using the model from O'Brien and Moldwin [2003]. We used the model from Shue et al. [1998] and adapted by Herrera et al. [2016] to determine the position of the magnetopause. For each event, we kept the minimal values of the positions of the magnetopause and the plasmapause during the time ranges corresponding to the crossing of the structures. We also made the distinction between the CIRs (in blue) and the ICMEs (in red).
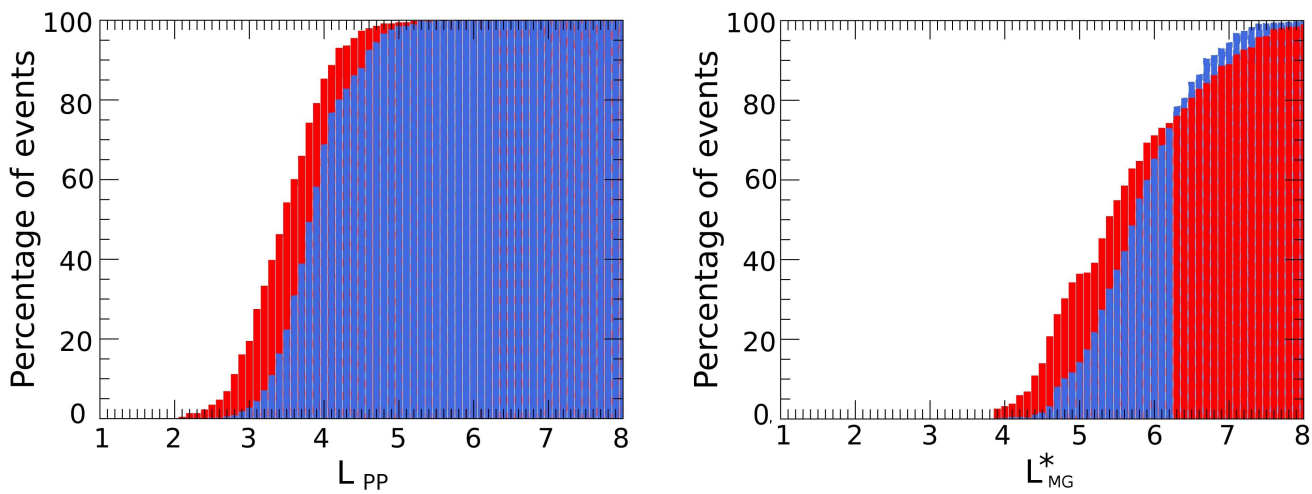

Figure 5. Sum curve of the postion of the plasmapause (left panel) and the magnetopause (right panel) depending on the solar wind structure : ICMEs (in red) and CIRs (in blue).

The left panel shows that ICMEs can push the plasmapause at $L^{*}$ as low as $L^{*}=2$. In the other hand, no CIR in our list of events can push the plasmapause to $L^{*}<3$. Concerning the balance between accelerations and loss processes at low $L^{*}$, one can therefore expect that accelerations processes will dominate at lower $L^{*}$ for ICMEs than CIRs. This can be observed on figure 1, notably for $>1 \mathrm{MeV}$ electrons. For lower energy channels, there is still a difference between CIRs and ICMEs but much weaker. For these energies, 
the position of the plasmapause remains the same, the pitch angle scattering is much less efficient. Therefore, the limit between loss and acceleration processes will be found at lower $L^{*}$ for these energies. This explains why CIRs can increase the flux at $L^{*}$ for the lowest energy channels but not for $>1 \mathrm{MeV}$.

On the right panel, we can see that ICMEs also compress the magnetopause to lower $L$ than CIRs during the events. This indicate that the loss process by magnetopause shadowing is more efficient during the former type of event. On the figure 1, it can be seen that, indeed, ICMEs cause more decreases of the flux than CIRs for high values of $L$ (at least for $>30 \mathrm{keV}$ and $>100 \mathrm{keV}$ electrons).

The consideration of the physical processes also allows to qualitatively explain the results of figure 3 for the low values of $L^{*}$. The intensity of magnetic storm also affect the balance between acceleration and loss processes. The more intense is the magnetic storm and the more efficient is the radial diffusion and the more restricted is the plasmasphere. Therefore, it is expected that acceleration processes will dominate at lower $L^{*}$ for more intense magnetic storms as it is observed on figure 3 .

\section{Conclusions}

We studied the variations of electron fluxes in the radiation belts due to the interaction of solar wind structures with the magnetosphere. We benefited from the NOAAPOES data which provide data on the full range of $L^{*}$ a great time range. Furthermore, by using several lists of events, we made the distinction between CIRs and ICMEs

Our study emphasized the strong dependency of these reactions to the considered energy, the $L *$ value and the type of structure. For ICMEs, one can subdivide the belts in two regions: in the inner part $\left(L^{*} \approx 2-4.5\right)$ the ICMEs will trend to increase the flux while in the outer part $\left(L^{*}>4.5\right)$, most of the ICMEs will decrease the flux. This is true for all energy channels despite the percentage of events making the flux increase in the inner part is significantly lower for $>1 \mathrm{MeV}$ electrons than for other energy channels. In the other hand, CIR have much less access to the innermost part of the belts. Therefore, the cause almost no reaction at $L^{*}<2.5$ for the lowest energy channels and even for $L^{*}<$ 3.5 for $>1 \mathrm{MeV}$ electrons. Then, most of them increase the flux on a large range of $L^{*}$ and only a limited number decrease the flux (especially for low energies). 
It was also found that the maximum amplitude of increase $(R>100)$ are found in a limited range of $L^{*}$ (around $L^{*} \approx 3$ ). At this deepness both CIRs and ICMEs can increase the flux for low energy channels while only ICMEs can cause such variations for the highest energies.

Lastly, we enlighten the different reactions of the belts depending on the intensity of the related magnetic storms. It appears clearly that the percentage of events that increase the flux increase with the intensity of the storm. In addition, strong magnetic storm have access to much inner part of the belts.

One strong challenge for the future, especially for space weather purpose will be to determine some pertinent lists of events from the radiation belts point of view. To do so, one should be able to determine the characteristics of the events that lead to an increase of the flux. It is clear that these characteristics will not be the same depending on the energy and $L^{*}$ as we showed, for example, that the amplitude of the flux variations and the intensity of the magnetic storms are linked for low values of $L^{*}$ but not in the external shells.

One could also consider the efficient events as those able to increase the flux above a threshold (rather than the relative variations). This has been the subject of a parallel study than will be the purpose of a future publication.

\section{Acknowledgments}

We are thankful to the NOAA-POES for online data access. The data are available on the CDAweb at http://cdaweb.gsfc.nasa.gov/. The lists of events come from the previous studies of Jian et al. [2006],Mitsakou and Moussas [2014], and Kilpua et al. [2015]. Rémi Benacquista is thankful for funding from CNES and ONERA. R.B thanks the two anonymous referees for help in evaluating this paper.

\section{References}

Alves, M. V., E. Echer, and W. D. Gonzalez (2006), Geoeffectiveness of corotating interaction regions as measured by Dst index, Journal of Geophysical Research, 111(A7), doi:10.1029/2005JA011379.

Anderson, B. R., R. M. Millan, G. D. Reeves, and R. H. W. Friedel (2015), Acceleration and loss of relativistic electrons during small geomagnetic storms: ELECTRON FLUX RESPONSE TO SMALL STORMS, Geophysical Research Letters, 42(23), 10,113- 
10,119, doi:10.1002/2015GL066376.

Benacquista, R., S. Rochel, and G. Rolland (2017), Understanding the variability of magnetic storms caused by ICMEs, Annales Geophysicae, 35(1), 147-159, doi: 10.5194/angeo-35-147-2017.

Borovsky, J. E., and M. H. Denton (2006), Differences between CME-driven storms and CIR-driven storms, Journal of Geophysical Research, 111(A7), doi: 10.1029/2005JA011447.

Cannon, P. (2013), Extreme space weather: impacts on engineered systems and infrastructure., Royal Academy of Engineering, oCLC: 830024325.

Herrera, D., V. F. Maget, and A. Sicard-Piet (2016), Characterizing magnetopause shadowing effects in the outer electron radiation belt during geomagnetic storms: CHARACTERIZING MAGNETOPAUSE SHADOWING, Journal of Geophysical Research: Space Physics, 121(10), 9517-9530, doi:10.1002/2016JA022825.

Hietala, H., E. K. J. Kilpua, D. L. Turner, and V. Angelopoulos (2014), Depleting effects of ICME-driven sheath regions on the outer electron radiation belt, Geophysical Research Letters, 41(7), 2258-2265, doi:10.1002/2014GL059551.

Jian, L., C. T. Russell, J. G. Luhmann, and R. M. Skoug (2006), Properties of Stream Interactions at One AU During 1995 - 2004, Solar Physics, 239(1-2), 337-392, doi: 10.1007/s11207-006-0132-3.

Kataoka, R., and Y. Miyoshi (2006), Flux enhancement of radiation belt electrons during geomagnetic storms driven by coronal mass ejections and corotating interaction regions: RADIATION BELT DURING CME/CIR STORMS, Space Weather, 4(9), n/a-n/a, doi: 10.1029/2005SW000211.

Kilpua, E. K. J., H. Hietala, D. L. Turner, H. E. J. Koskinen, T. I. Pulkkinen, J. V. Rodriguez, G. D. Reeves, S. G. Claudepierre, and H. E. Spence (2015), Unraveling the drivers of the storm time radiation belt response: RADIATION BELT AND STORM DRIVERS, Geophysical Research Letters, 42(9), 3076-3084, doi: 10.1002/2015GL063542.

Mitsakou, E., and X. Moussas (2014), Statistical Study of ICMEs and Their Sheaths During Solar Cycle 23 (1996 - 2008), Solar Physics, 289(8), 3137-3157, doi: 10.1007/s11207-014-0505-y.

Miyoshi, Y., and R. Kataoka (2005), Ring current ions and radiation belt electrons during geomagnetic storms driven by coronal mass ejections and corotating interaction regions, 
Geophysical Research Letters, 32(21), doi:10.1029/2005GL024590.

Miyoshi, Y., and R. Kataoka (2008), Flux enhancement of the outer radiation belt electrons after the arrival of stream interaction regions: RADIATION BELT RESPONSE TO SIR, Journal of Geophysical Research: Space Physics, 113(A3), n/a-n/a, doi: 10.1029/2007JA012506.

Miyoshi, Y., R. Kataoka, Y. Kasahara, A. Kumamoto, T. Nagai, and M. F. Thomsen (2013), High-speed solar wind with southward interplanetary magnetic field causes relativistic electron flux enhancement of the outer radiation belt via enhanced condition of whistler waves: SOLAR WIND-RADIATION BELT COUPLING, Geophysical Research Letters, 40(17), 4520-4525, doi:10.1002/grl.50916.

O’Brien, T. P., and M. B. Moldwin (2003), Empirical plasmapause models from magnetic indices: EMPIRICAL PLASMAPAUSE MODELS, Geophysical Research Letters, 30(4), doi:10.1029/2002GL016007.

Reeves, G. D., K. L. McAdams, R. H. W. Friedel, and T. P. O'Brien (2003), Acceleration and loss of relativistic electrons during geomagnetic storms: ACCELERATION AND LOSS OF RELATIVISTIC ELECTRONS, Geophysical Research Letters, 30(10), n/an/a, doi:10.1029/2002GL016513.

Reeves, G. D., R. H. W. Friedel, B. A. Larsen, R. M. Skoug, H. O. Funsten, S. G. Claudepierre, J. F. Fennell, D. L. Turner, M. H. Denton, H. E. Spence, J. B. Blake, and D. N. Baker (2016), Energy-dependent dynamics of $\mathrm{keV}$ to $\mathrm{MeV}$ electrons in the inner zone, outer zone, and slot regions: ENERGY-DEPENDENT RADIATION BELT DYNAMICS, Journal of Geophysical Research: Space Physics, 121(1), 397-412, doi: 10.1002/2015JA021569.

Richardson, I. G., and H. V. Cane (2011), Geoeffectiveness ( Dst and $K p$ ) of interplanetary coronal mass ejections during 1995-2009 and implications for storm forecasting: GEOEFFECTIVENESS OF ICMES, Space Weather, 9(7), n/a-n/a, doi: 10.1029/2011SW000670.

Shue, J.-H., P. Song, C. T. Russell, J. T. Steinberg, J. K. Chao, G. Zastenker, O. L. Vaisberg, S. Kokubun, H. J. Singer, T. R. Detman, and H. Kawano (1998), Magnetopause location under extreme solar wind conditions, Journal of Geophysical Research: Space Physics, 103(A8), 17,691-17,700, doi:10.1029/98JA01103.

Su, Z., F. Xiao, H. Zheng, Z. He, H. Zhu, M. Zhang, C. Shen, Y. Wang, S. Wang, C. A. Kletzing, W. S. Kurth, G. B. Hospodarsky, H. E. Spence, G. D. Reeves, H. O. Funsten, 
J. B. Blake, and D. N. Baker (2014), Nonstorm time dynamics of electron radiation belts observed by the Van Allen Probes, Geophysical Research Letters, 41(2), 229-235, doi:10.1002/2013GL058912.

Su, Z., H. Zhu, F. Xiao, Q.-G. Zong, X.-Z. Zhou, H. Zheng, Y. Wang, S. Wang, Y.-X. Hao, Z. Gao, Z. He, D. N. Baker, H. E. Spence, G. D. Reeves, J. B. Blake, and J. R. Wygant (2015), Ultra-low-frequency wave-driven diffusion of radiation belt relativistic electrons, Nature Communications, 6, 10,096, doi:10.1038/ncomms10096.

Su, Z., Z. Gao, H. Zhu, W. Li, H. Zheng, Y. Wang, S. Wang, H. E. Spence, G. D. Reeves, D. N. Baker, J. B. Blake, H. O. Funsten, and J. R. Wygant (2016), Nonstorm time dropout of radiation belt electron fluxes on 24 September 2013: NONSTORM RADIATION BELT DROPOUT, Journal of Geophysical Research: Space Physics, 121(7), 6400-6416, doi:10.1002/2016JA022546.

Turner, D. L., T. P. O’Brien, J. F. Fennell, S. G. Claudepierre, J. B. Blake, E. K. J. Kilpua, and H. Hietala (2015), The effects of geomagnetic storms on electrons in Earth's radiation belts: RADIATION BELT ELECTRON RESPONSES TO STORMS, Geophysical Research Letters, 42(21), 9176-9184, doi:10.1002/2015GL064747.

Turner, N. E., E. J. Mitchell, D. J. Knipp, and B. A. Emery (2006), Energetics of magnetic storms driven by corotating interaction regions: A study of geoeffectiveness, in Geophysical Monograph Series, vol. 167, edited by B. Tsurutani, R. McPherron, W. Gonzalez, G. Lu, J. H. A. Sobral, and N. Gopalswamy, pp. 113-124, American Geophysical Union, Washington, D. C., dOI: 10.1029/167GM11.

Yando, K., R. M. Millan, J. C. Green, and D. S. Evans (2011), A Monte Carlo simulation of the NOAA POES Medium Energy Proton and Electron Detector instrument: TECHNIQUE, Journal of Geophysical Research: Space Physics, 116(A10), n/a-n/a, doi: 10.1029/2011JA016671. 